\title{
Estado periodontal y de la mucosa oral en un grupo de embarazadas: Estudio clínico
}

\author{
Periodontal and mucosal status in a group of pregnant women: Clinical \\ study
}

\author{
LACALZADA-PASTOR IM* \\ GIL-SAMANIEGO J* \\ GIMÉNEZ-JUNCOSA IM* \\ LÓPEZ-LÓPEZ J** \\ CHIMENOS-KÜSTNER E**
}

\begin{abstract}
Lacalzada-Pastor M, Gil-Samaniego J, Giménez-Juncosa M, LópezLópez J, Chimenos-Küstner E. Estado periodontal y de la mucosa oral en un grupo de embarazadas: Estudio clínico. Av Periodon Implantol. 2011; 23, 2: 123-128.
\end{abstract}

\section{RESUMEN}

Introducción: Los cambios hormonales en el embarazo se reflejan en la salud periodontal de las pacientes, se pretende cuantificar y evaluar estos cambios en la cavidad oral de 165 pacientes embarazadas, así como el estado de su mucosa oral y relacionar su percepción de salud oral con los resultados de su estado periodontal.

Métodos: Pacientes de revisión de rutina de un centro de atención primaria. Se les realiza una exploración donde se evalúa el estado periodontal, mediante el Índice de Necesidad de Tratamiento Periodontal de la Comunidad (C.P.I.T.N.), y el estado de las mucosas. Los dientes escogidos para el sondaje fueron el 16, 11,26,36,31 y 46 de cada paciente y se tomó como valor representativo el más alto de todos los sextantes. 56 acudieron para realizarse una reevaluación.

Resultados: De las 165 embarazadas el 5\% (9) fueron consideradas invalorables. El 35\% dieron como resultado valor 0 (57); el $46 \%$ valor 1 (76); el $12 \%$ valor 2 (20); y el $2 \%$ valor 3 (1). De las 56 que acudieron a la reevaluación el 23\% (13) mejoraron, el 10\% (6) empeoraron y el 66\% (37) se mantuvieron. De las 165 sólo una presentó una lesión en mucosas, una ránula.

Conclusiones: Aunque hubo un porcentaje sin patología periodontal, 35\%, la mayoría padecía gingivitis $(46 \%)$ y algunas periodontitis (14\%). Por tanto consideramos importante recomendar a las pacientes embarazadas una exploración bucodental y realizar una labor preventiva en todas las pacientes en edad fértil con el fin de evitar consecuencias negativas.

PALABRAS CLAVE: Gingivitis, periodontitis, embarazo, hormonas, mucosa oral.

\section{SUMMMARY}

Background: The hormonal changes during pregnancy are reflected in the periodontal health, the aim of this study is to evaluate and quantify these changes in the oral cavity of 165 patients as well as their mucosal status and to compare the perception pregnant women have of their own oral health and the obtained results of their periodontal status.

Methods: Patients included in the study were selected from the routine dental revision program of a public health centre. An oral exploration was performed. Periodontal status was evaluated by means of the Community Periodontal Index of Treatment Needs (CPITN). Teeth 16, 11, 26, 36, 31 and 46 from each patient were proben and the chosen value was the highest one of all sextants. 56 patients came back for reevaluation. The status of the oral mucosa was also inspected.

Results: Among the 165 patients, the 5\% were considered non-valuable (9), the $35 \%$ scored value 0 (57), the $46 \%$ scored value 1 (76), the $12 \%$ value 2 (20) and the $2 \%$ value 3 (1). 
Among the 56 who came back for reevaluation 13 of them did improve (23\%), 6 got worse (10\%) and 37 (66\%) maintained. Only one person had an oral lesion: a ranula.

Conclusions: Despite there was a group free of periodontal pathology, $35 \%$, the majority of the patients $(46 \%)$ had gingivitis and some of them periodontitis (14\%). It is important for us to recommend the pregnant patients to come for a buccal exploration as well as carrying out a preventive labour in all patients in fertile age.

KEY WORDS: Gingivitis, periodontitis, pregnancy, hormones, oral mucosa.

Fecha de recepción: 20 de noviembre de 2009.

Fecha de aceptación: 22 de diciembre de 2009.

\section{INTRODUCCIÓN}

Según la Asociación Dental Americana (ADA) existen dos mitos con respecto al embarazo y la salud oral: $\mathrm{La}$ pérdida de dientes como consecuencia de éste y la obtención de calcio de los dientes si la dieta es insuficiente para proporcionar todo el calcio requerido (primacía ovular). La principal causa de la enfermedad periodontal es la placa bacteriana (y no las hormonas), por lo que se recomiendan tartrectomías en el segundo y tercer trimestre de embarazo para controlar la gingivitis. Los tratamientos odontológicos pueden y deben hacerse, evitando el primer trimestre (1).

A nivel gingival existen receptores de estrógenos y progesterona. Estas hormonas provocan alteraciones vasculares, celulares, microbiológicas e inmunológicas. Sin embargo, en ausencia de placa estos cambios no dan lugar a enfermedad periodontal (2).

Sin duda, una de las funciones más importantes de las hormonas ováricas es la preparación de las condiciones necesarias para el embarazo. Cuando el óvulo es fecundado, el cuerpo lúteo del ovario no degenera, sino que va creciendo y secretando estrógeno y progesterona.

Durante los últimos tres meses de gravidez, los niveles de estrógenos y progesterona descienden y aumenta la secreción de otras hormonas, como la relaxina, que tiene por función relajar la pelvis y ablandar el cuello del útero; y la oxitocina, que estimula la contracción del útero. Tras el nacimiento, la prolactina interviene en la secreción de leche y la oxitocina en su eyección.

La tasa de secreción de estrógenos en el embarazo es muy superior a la secretada en estado no gravídico. El principal estrógeno secretado por el folículo en desa- rrollo es el 17 ß-estradiol, esta hormona aumenta hasta 100 veces, y los niveles circulantes de otras son incluso mayores. La tasa de secreción de la progesterona en los últimos meses de embarazo alcanza un ritmo de 250-350 mg/día, cuando en la fase luteínica del ciclo menstrual se secretan 20 mg/día (3).

El embarazo no es un factor decisivo en la aparición de gingivitis; sin embargo, hay un incremento en la incidencia de ésta. Aunque la incidencia de la periodontitis no se vea aumentada algunos estudios avalan que la severidad de la periodontitis es mayor cuando aparece durante el embarazo (4). Algunos autores describen un aumento de la movilidad dentaria durante la gestación (4). Por otro lado, numerosos estudios relacionan la periodontitis con partos prematuros y niños de bajo peso al nacer (5-8).

Actualmente son pocos los trabajos publicados sobre las alteraciones prevalentes en la cavidad bucal durante el embarazo (4). Díaz-Guzmán y cols. realizaron un estudio prospectivo, observacional y transversal en la Universidad de La Salle, México, donde se comparó la prevalencia de lesiones en un grupo de embarazadas y en un grupo control obteniendo un resultado similar, siendo el leucoedema y la úlcera traumática las lesiones más prevalentes. Únicamente se registró un ligero aumento de la incidencia del granuloma piógeno y de la glositis migratoria benigna en el grupo de gestantes (4).

Palacios Sánchez y cols. realizaron una revisión bibliográfica donde determinaron que el $5 \%$ de las gestantes presentan un granuloma piógeno durante el embarazo, éste suele aparecer en el primer o segundo mes y remitir tras el parto (2).

Siendo el embarazo un estado con unas condiciones propias diferentes creemos necesario estudiar cuáles 
son los cambios producidos en la cavidad bucal y qué lesiones aparecen como consecuencia del mismo.

Por todo esto nos pareció importante hacer un estudio piloto que evaluara el estado periodontal durante la gestación.

\section{MATERIAL Y MÉTODOS}

Las exploraciones bucodentales fueron realizadas en un centro de salud (Centro de salud Jaume I de Vilanova y la Geltrú, Barcelona), durante el periodo comprendido entre el 1 de Julio del 2008 y el 29 de Diciembre del 2008.

Se estudió una población de 165 pacientes embarazadas remitidas desde el servicio de ginecología del mismo centro. Los criterios de selección fueron pacientes embarazadas que estuvieran en el primer trimestre de gestación y que fueran a llevar a término el embarazo en esa misma población para poder realizarles el posterior seguimiento.

El tiempo de estudio fue de seis meses. A cada paciente se le realizó la revisión al final de su primer trimestre de embarazo y se le propuso una reevaluación en el tercero.

El protocolo seguido para cada exploración fue el siguiente: Examen del estado periodontal y de las mucosas. La evaluación del estado periodontal se realizó mediante el Índice de Necesidad de Tratamiento Periodontal de la Comunidad (C.P.I.T.N.) debido a su eficacia como herramienta diagnóstica y su universalidad en estudios epidemiológicos periodontales. La sonda periodontal propuesta por la OMS fue escogida como instrumento diagnóstico para la valoración clínica de la destrucción de los tejidos periodontales, con ella se midieron la profundidad de las bolsas periodontales y la pérdida de inserción tomando como referencia la línea amelocementaria.

Se dividió la boca en sextantes y se sondaron los dientes $16,11,26,36,31$ y 46 , tomando como valor representativo el más alto de todos los sextantes. Se excluyeron las pacientes en las cuales el $50 \%$ o más de los dientes a sondar se encontraran ausentes.

La valoración de la destrucción periodontal fue medida con la escala del C.P.I.T.N, resumida en la tabla 1. La técnica de sondaje consistió en insertar suavemente la sonda en el surco gingival, con una presión de 20

\section{TABLA 1.- ESCALA C.P.I.T.N.}

\begin{tabular}{|ll|}
\hline Código 0 & Periodonto sano. \\
\hline Código 1 & $\begin{array}{l}\text { Sangrado inmediato o hasta } 30 \text { seg. des- } \\
\text { pués del sondaje. }\end{array}$ \\
\hline Código 2 & $\begin{array}{l}\text { Presencia de cálculo u obturaciones des- } \\
\text { bordantes. }\end{array}$ \\
\hline Código 3 & Bolsas periodontales de 3,5 a $5,5 \mathrm{~mm}$. \\
\hline
\end{tabular}

a 25 gramos, para lo cual se realizaron ensayos previos. La inserción fue paralela al eje vertical del diente y se deslizó alrededor de todas las superficies dentales, sondando por vestibular y lingual en mesial, distal y el centro de las caras de cada diente.

Para la valoración de la percepción de salud oral se utilizó una escala visual analógica donde 0 era considerar una salud pésima y 10 una salud óptima.

A las pacientes que presentaban cálculo se les realizó una tartrectomía en el segundo trimestre para intentar minimizar los efectos de la placa.

\section{RESULTADOS}

El rango de edad de las pacientes comprendía de los 17 a los 40 años.

Los resultados de la primera exploración periodontal (Tabla 2) indican que 9 pacientes fueron consideradas invalorables (5\%), ya que el $50 \%$ o más de los dientes a sondar estaban ausentes; 57 , dieron como resultado valor $0(35 \%)$; 76 , valor 1 ( $46 \%) ; 20$, valor $2(12 \%)$ y 3 , valor $3(2 \%)$.

\section{TÄВА 2 - ESTĀDO PERIODONTAL AL INICIO DEL ESTUDIO, PRIMER TRIMESTRE DE GESTÁCIÓN}

\begin{tabular}{|c|c|}
\hline Valor $0 .$. & $57(35 \%)$ \\
\hline Valor 1 . & $76(46 \%)$ \\
\hline Valor 2 & $20(12 \%)$ \\
\hline Valor $3 .$. & $3(2 \%)$ \\
\hline Invalorables ............... & $9(5 \%)$ \\
\hline
\end{tabular}


De las 165 pacientes que acudieron a la primera visita, 56 volvieron para realizar una reevaluación en su tercer trimestre $(34 \%)$. De todas ellas sólo una presentaba una lesión en la mucosa, una ránula (Figura 1).

Los resultados de la reevaluación, al final del tercer trimestre del embarazo, se resumen en la tabla 3, 20 pacientes dieron como resultado valor 0 (36\%); 24 valor 1 (42\%); 11 valor $2(20 \%)$ y 1 valor $3(2 \%)$.

En las figuras 2 y 3 se reflejan los resultados al inicio del estudio y en la reevaluación. 13 pacientes habían mejorado (23\%); 6 empeorado (10\%) y 37 se mantenían (66\%). Haciendo una comparativa porcentual se observa que el grupo de mayor población tanto en la primera visita como en la reevaluación es el de pacientes que presentan gingivitis (valor 1 ).

El 85\% de las pacientes que acudieron a la reevaluación percibían que tenían mejor salud bucodental que al principio del estudio cuando la realidad era que el

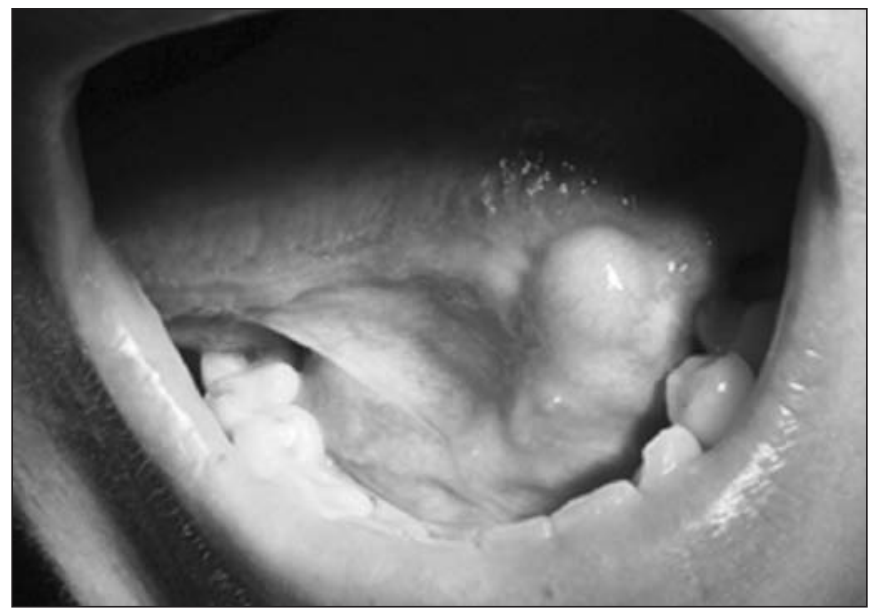

Fig. 1: Ránula en una paciente embarazada de 2 meses y medio.

\begin{tabular}{|c|c|}
\hline $\begin{array}{r}\text { TABLA 3.- RESULTADOS } \\
\text { LA REEVALUACIÓN, AL FIN } \\
\text { TERCER TRIMESTRE DEL EM }\end{array}$ & $\begin{array}{l}\text { E } \\
\text { DEL } \\
\text { ARAZO }\end{array}$ \\
\hline Valor $0 \ldots$. & $20(36 \%)$ \\
\hline Valor $1 \ldots . . .$. & $24(42 \%)$ \\
\hline Valor $2 \ldots .$. & $11(20 \%)$ \\
\hline Valor $3 \ldots . .$. & $1(2 \%)$ \\
\hline
\end{tabular}

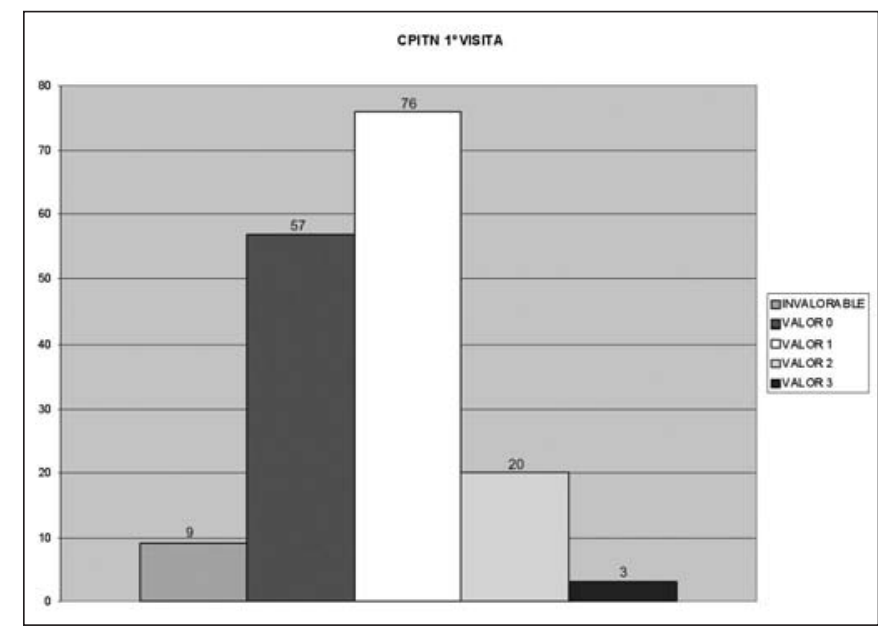

Fig. 2: Estado periodontal en primer trimestre de embarazo.

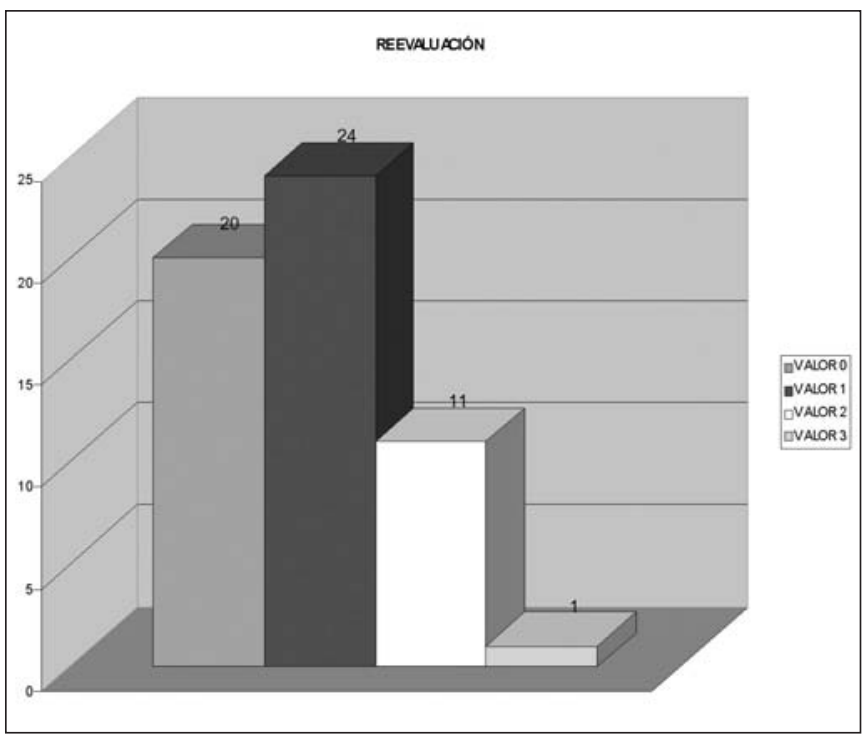

Fig. 3: Estado periodontal en tercer trimestre, reevaluación.

10\% tenía peor, el $37 \%$ se mantenía igual y sólo el $23 \%$ había mejorado.

\section{DISCUSIÓN}

Para algunos autores (5-9) no existen suficientes indicios que demuestren la relación causal entre la gingivitis en el embarazo y el desarrollo de la periodontitis.

Por un lado el aumento de vascularización que se produce en el embarazo podría ser la causa del sangrado 
gingival que perciben la mayoría de embarazadas. Por otro, el embarazo per se no causa gingivitis: es necesaria la presencia de placa bacteriana (4). Sin embargo, aunque las hormonas no sean el único factor decisivo ni el más relevante para causar inflamación gingival, en nuestro estudio la mayoría de las pacientes presentaban gingivitis (46\%), lo que nos sugiere una asociación entre la gestación y la patología periodontal.

Debido a que el embarazo es una situación fisiológica especial en la que se debe ser especialmente cauto y conservador en las técnicas empleadas harían falta más estudios que nos aseguraran que el tratamiento periodontal es beneficioso para la futura madre y seguro para el feto. La realidad a día de hoy es que existe una gran controversia en este sentido $(5,10,11)$. Hay estudios que demuestran la asociación entre la enfermedad periodontal y los partos prematuros y los niños de bajo peso al nacer. Lo que no es tan evidente hasta la fecha es si el tratamiento periodontal reduce o no la tasa de incidencia, ya que para algunos autores el tratamiento periodontal realizado durante el embarazo reduce dicha incidencia $(5,10)$, mientras que para otros no (11).

Algunos estudios avalan que la utilización de terapia periodontal no quirúrgica durante el segundo trimestre de gestación es segura y efectiva, así como otros tratamientos odontológicos conservadores (6).

Estudios recientes demuestran que la salud bucodental y la calidad de vida relacionada con la misma es más pobre en las pacientes embarazadas que en las no gestantes (12).

En nuestro estudio las pacientes de la reevaluación manifestaban encontrarse mejor cuando se les preguntaba por su estado bucal, aunque la mayoría de ellas se mantenían en el mismo estado periodontal y algunas incluso habían empeorado. El 85\% afirmaba encontrarse mejor, cuando la realidad era que sólo el $23 \%$ había mejorado, el 10\% había empeorado y el $66 \%$ se mantenía como al principio. Este dato coincide con los estudios que determinan que los programas de prevención ayudan a mejorar la calidad de vida de las gestantes y a mejorar su percepción (13). Estos resultados de sensación de bienestar se obtuvieron en la mayoría sólo por el hecho de realizarles en la primera visita una explicación de técnicas de cepillado, técnica de Bass modificada, y asesoramiento mediante consejos higiénicos y dietéticos. La percepción de salud oral en las embarazadas se consigue mejorar mediante asesoramiento sobre técnicas de higiene y control en el embarazo.
En un estudio llevado a cabo en la Universidad de Carolina del Norte por Wilder R, Robinson C, Jared HL, Lieff S y Boggess K. se pudo observar que el $49 \%$ de los ginecólogos nunca o rara vez recomendaban la exploración bucodental, y al examinar los conocimientos de los médicos sobre la periodontitis y sus riesgos para el embarazo así como el asesoramiento hacia las pacientes los resultados fueron bastante desalentadores: el $22 \%$ de los médicos revisaban a las embarazadas prenatalmente, el $9 \%$ las revisaba periódicamente y el $48 \%$ sólo cuando la paciente mencionaba algún problema (14).

En nuestro estudio estos resultados fueron distintos ya que el $100 \%$ de las pacientes fueron remitidas desde el servicio de ginecología, sin embargo los resultados anteriores sugieren la necesidad de realizar una labor preventiva e informativa sobre la periodontitis y cómo afecta ésta a las pacientes durante el embarazo.

Es fundamental informar a las pacientes de la importancia de la exploración bucodental durante el embarazo para poder diagnosticar precozmente y tratar los posibles problemas. Hay que informar no sólo a las pacientes, sino también a los profesionales de la salud que estén en contacto con ellas para que puedan asesorarlas y derivarlas a un servicio de odontología. Debemos aumentar las medidas preventivas (enseñanza de técnicas de higiene oral, tartrectomías, complementos del cepillado etc.) en las mujeres de edad fértil, para minimizar las posibles consecuencias.

Es difícil establecer unos protocolos de actuación pero las estadísticas nos obligan a tomar partido, la inflamación gingival tiene una prevalencia del 50 al 98\% en la gestación según algunos autores (4) y del 30 al $70 \%$ según otros (15). En nuestro estudio la prevalencia fue del 42 al $46 \%$, en cualquier caso cifras nada desdeñables.

La lesión bucal asociada más frecuentemente al embarazo es el granuloma piógeno, con una incidencia del $5 \%$ en las gestantes (2) sin embargo en nuestro estudio no hubo ninguna paciente que lo presentara. La única lesión encontrada fue una ránula, con una prevalencia del 0,6\%.

\section{BIBLIOGRAFÍA}

1. Lauer CD, Turp JC. Without risks and side effects? Product advertisements in dental journals. Schweiz Monatsschr Zahnmed 2006;116(7):718-24. 
2. Palacios-Sánchez B, Cerero-Lapiedra R, Campo-Trapero J, Esparza-Gómez G. Alteraciones gingivales no relacionadas con placa. RCOE 2006; 11 (1):43-55.

3. Pocock G, Richards C D. Fisiología humana. La base de la medicina. Masson: Barcelona; 2005.

4. Díaz-Guzmán LM, Castellanos-Suárez JL. Lesiones de la mucosa bucal y comportamiento de la enfermedad periodontal en embarazadas. Med Oral Patol Oral Cir Bucal 2004;9(5):430-7.

5. Radnai M, Pál A, Novák T, Urbán E, Eller J, Gorzó I. Benefits of periodontal therapy when preterm birth threatens. J Dent Res 2009;88(3):280.

6. Silk H, Douglass AB, Douglass JM, Silk L. Oral health during pregnancy. Am Fam Physician 2008;77(8):1139-44.

7. Bobetsis YA, Barros SP, Offenbacher S. Exploring the relationship between periodontal disease and pregnancy complications. J Am Dent Assoc 2008;139 (3):252.

8. Offenbacher S, Lin D, Strauss R, Mckaig R, Irving J, Barros SP, Moss K, Barrow DA, Hefti A, Beck JD. Effects of periodontal therapy during pregnancy on periodontal status, biologic parameters, and pregnancy outcomes: a pilot study. J Periodontol 2006;77(12):2011-24.

9. Gürsoy M, Pajukanta R, Sorsa T, Könönen E.Clinical changes in periodontium during pregnancy and postpartum. J Clin Periodontol 2008; 35(7):576-83.

10. Radnai M, Pál A, Novák T, Urbán E, Eller J, Gorzó I. The possible effect of basic periodontal treatment on the outcome of pregnancy. Fogorv Sz 2008;101(5):179-85.

11. Wimmer G, Pihlstrom BL. A critical assessment of adverse pregnancy outcome and periodontal disease. J Clin Periodontol 2008;35(8):380-97.
12. Acharya S, Bhat PV. Oral-Health-Related Quality of life during pregnancy. J Public Health Dent 2009;69(2):74-7.

13. Acharya S, Bhat PV, Acharya S. Factors affecting oral health-related quality of life among pregnant women. Int J Dent Hyg. 2009;7(2):102-7.

14. Wilder R, Robinson C, Jared HL, Lieff S, Boggess K. Obstetricians knowledge and practice behaviors concerning periodontal health and preterm delivery and low birth weight. J Dent Hyg 2007;81(4):81.

15. Barak S, Oettinger-Barak O, Oettinger M, Machtei EE, Peled M, Ohel G. Common oral manifestations during pregnancy: a review. Obstet Gynecol Surv 2003;58(9): 624-8.

16. Russell SL, Mayberry LJ. Pregnancy and oral health: a review and recommendations to reduce gaps in practice and research. MCN Am J Matern Child Nurs 2008;33(1): 32-7.

17. Vasiliauskiene I, Milciuviene S, Bendoraitiene E, Narbutaite J, Slabsinskiene E, Andruskeviciene V. Dynamics of pregnant women's oral health status during preventive programme. Stomatologija 2007;9(4):129-36.

18. Plutzer K, Spencer AJ. Efficacy of an oral health promotion intervention in the prevention of early childhood caries. Community Dent Oral Epidemiol 2008;36(4):335-46.

19. Jafarzadeh H, Sanatkhani M, Mohtasham. Oral pyogenic granuloma: a review. N J Oral Sci 2006;48(4):167-75.

\section{CORRESPONDENCIA}

\section{María Lacalzada Pastor}

E-mail: marialacalzadapastor@hotmail.com 